generally accepted theory of aetiology. Most of my cases appeared to be infective in origin, and two of them followed instrumentation. It does appear that the disease is associated with poor hygiene. Fournier's gangrene was fairly common in Europe and North America at the turn of the century but is now rarely reported from these areas. More recent reports of more than one case have come from areas with poor living standards. - $6^{-6}$

It was suggested that the patient described may have had an increased tendency to vascular insufficiency because of a high titre of cold haemagglutinins in his serum. It is possible that this and the trauma he suffered were enough to precipitate gangrene of the scrotum by vascular thrombosis. Several au'hors have proposed that the cause of Fournier's gangrene is an arterial thrombosis precipitated by an infection, analogous to cavernous sinus thrombosis. ${ }^{6-8}$ This case could be compatible with the thrombosis theory of aetiology.-I am, etc.,

P.A. Central Hospital,

Douglas J. Buchanan

Ndola, Zambia

1 Buchanan, D. J., Medical Fournal of Zambia, 2 Allen, J., Fournal of Cutaneous and GenitoUrinary Diseases, 1894, 12, 55.

3 Randall, A., Ұournal of Urology, 1920, 4, 219. $19,3,25,60$. 19J3, 25, 60. J., South African Medical fournal,
1951, 25, 222.

Sachdeva, Y. V., and Khanna, B. C., Indian Fournal of Surgery, 1949, 11, 178

Carver, J., British Fournal of Urology, 1939 ,

Mansfield, O. T., British fournal of Surgery, $1946,33,275$

\section{Asthma in the Elderly}

SIR,-May I briefly comment on two points in the paper by Drs. $H$. Y. Lee and $T$. B. Stretton (14 October, p. 93)?

Reversible obstruction of the airways occurs very often in both young and elderly patients suffering from chronic non-specific lung disease. The attacks, especially when repeated, are mostly accompanied by signs of alveolar hypoventilation. I feel that the 15 elderly patients studied by the authors fufilled only partly the criteria for the diagnosis of asthma-no hypercapnia and no reversibility of obstruction were found in most of them. Diminished vital capacity may indicate a restrictive ventilatory disturbance. I wonder if measurement of the $\mathrm{FEV}_{1}$ alone is suitable for the diagnosis of obstruction; if the residual volume is increased, a low $\mathrm{FEV}_{1}$ may simply result from high intrathoracic pressure during expiration. Now that body plethysmography is available, assessment of the residual volume and/or intrathoracic gas volume has become very simple, and the resistance of the smaller airways during normal expiration may also be calculated. I suggest therefore that the term "asthma" should be reserved for those cases in which reversible airways obstruction is quite clearly demonstrable and that it should not be used simply as a symptomatic label.

I also disagree with another statement of the authors concerning sputum eosinophilia. Our findings in more than 15,000 samples of bronchial secretion prepared from sputum by Mulder's technique during the past eight years have shown that eosinophilia is a very common consequence of bronchial hyperreactivity. Moreover, a longterm follow-up study in Europe's first model village for the treatment of chronic nonspecific lung disease in Zákànyszèk, South Hungary, showed bronchial eosinophilia to be fairly constant in the greater part of a total of 384 patients, only a few of whom ever had real obstructive attacks. Eosinophilia correlates closely with bacterial infection of the bronchi and may serve as a basic indicator in the assessment of airway infections, ${ }^{12}$ as active bronchial infection causes the eosinophils to disappear. Since the main physiological role attributed to eosinophils is the local release of histamine and since immune reactions of type I (reaginic type) often cause the production of eosinophil chemotactic factor, ${ }^{3}$ bronchial eosinophilia seems to be a very complex phenomenon. It is surely oversimplifying the position to say that "sputum eosinophilia is of considerable value in assessing asthmatic patients." It may be of value and it may not.-I am, etc.,

Department of Pneumology,

Miklos LaNYI Semmelweis University, Budapest

1 Lányi, M., Lancet, 1967, 1, 1165

2 Lányi, M., Deutsche Medizinische Wochenschrift, 3 Kay, A. B3, 2390 . Stechschulte, D. J., and Austen, K. F., fournal of Allergy, 197i, 47, 118.

SIR-I read with interest the article on this subject by Drs. H. Y. Lee and T. B. Stretton (14 October, p. 93). I am rather concerned, however, about the suggested treatment. While systemic corticosteroids are essential and life-saving at times their constant use has created over the past 10 years the problem of corticosteroid-dependent asthma. In my unit we have been trying over the past 18 months to treat these patients with initial doses of systemic corticosteroid followed by aerosol inhalants of beclomethasone. This is a synthetic, poorly-absorbing corticosteroid, and we have used it successfully in 82 out of 95 cases of late-onset asthma. These have been followed up for 12 months, and we hope to publish the results.-I am, etc.,

\section{S. S. Chatterjee}

Department of Respiratory Physiology, Baguley Hospital, Manchester

\section{Bicarbonate Solutions for Infusion}

SIR,-It has come to our attention that $1.4 \%$ sodium bicarbonate infusion solutions are no longer available commercially in the U.K. and that the lowest concentrations being produced regularly are $2.74 \%$ (Polyfusor-Boots) and $4 \cdot 2 \%$ (Travenol Laboratories-Baxter). These firms may produce $1.4 \%$ sodium bicarbonate as a special order but this often takes several months. It is therefore important that all hospitals which may at some time or another admit acutely poisoned patients should review their procedures for forced alkaline diuresis, as many of these regimens include $1.4 \%$ sodium bicarbonate solution.

Sodium bicarbonate solutions are used in forced alkaline diuresis regimens to promote the alkalinization of the urine and therefore it might appear that any procedure would be adequate which adjusts the proportion of sodium bicarbonate to the total infusion volume to give a urinary $\mathrm{pH}$ of 8 . (The $\mathrm{pH}$ indicator portion of Bililabstix is not suitable, in our experience, for this purpose and, if a $\mathrm{pH}$ meter is not available, narrow-range indicator paper should be used.) However, owing to the length of time required for the renal compensation of acid/base disturbances, the patient may become more alkalaemic than hitherto before producing a significantly alkaline urine if these more concentrated solutions are used. In our experience there may be a transient rise in the blood levels of phenobarbitone when the phenobarbitone-poisoned patient first develops a base excess, and we are concerned that this rise may be considerably higher if the rate of infusion of alkali in a forced alkaline diuresis regimen is increased at any time. It would appear to us that one method which would overcome this difficulty is to give the sodium bicarbonate simultaneously with other infusion fluids connected with a Y-piece, but we would be interested to hear what other cintres are planning to do.-We are, etc.,

E. SIMPSON M. J. STEWART Patricia GoOdHEad

Department of Clinical Chemistry,

Muryfield

Normal Range of "Effective Thyroxine Ratio"

SIR,-Standardized reagent kits for measurement of the "effective thyroxine ratio" (E.T.R.) have become avaulable relat.v.ly recently (Mallinckrodt U.K. Ltd.). This test has been shown ${ }^{12}$ to have a diagnostic accuracy comparable to that of a free thyroxine index in categorizing patients according to their thyroid status. Since the tentative normal range $(0 \cdot 86-1 \cdot 13)$ published by the manufacturers was based upon a population of wide geographical distribution, users were recommended to establish their own normal values. Having used this test routinely in our biochemistry department for some months, we have now analysed our first 1,000 results with this object in view, and present our findings here for the interest of other current or potential users.

We used the reagent kits according to the manufacturers' instructions, except that a pool of at least 50 human sera taken from routine samples was used as a standard instead of the lyophilized material supplied; this modification was found to improve the precision of the assay (from S.D. $=0.032$ to S.D. $=0.019$ within batch)

The results, plotted on probability paper according to Hoffmann ${ }^{3}$ are shown in the Figure, where it can be seen that the cumulative percentage incidence of the values found is linear between approximately 10 to $85 \%$. It seems reasonable to assume that this range of intermediate values, containing some $75 \%$ of all the results, is derived from euthyroid subjects and that, since the linearity of the graph in this region indicates a Gaussian distribution, the range of E.T.R. values for $95 \%$ of euthyroid subjects may be obtained by extrapolation of this straight line as suggested by Hoffmann. ${ }^{3}$ The range thus found is $0.88-1 \cdot 09$. This is similar to that found by Thorson et al. ${ }^{1}$ in 198 normal subjects (0.89-1.09), but broader than that found by Murray et al. ${ }^{2}$ in 80 such subjects $(0.89$ 1.05). The latter authors suggested the existence of "borderline" zones between 0.86 and 0.89 and between 1.06 and 1.09; this is consistent with our own findings, as shown in the Figure, where deviation from 